\title{
Assessing the Prediction Effect of Various Prognosis Model for 28-Day Mortality in Acute-on-Chronic Liver Failure Patients
}

This article was published in the following Dove Press journal: Risk Management and Healthcare Policy

\author{
Lin Xiang Liu* \\ Yue Zhang* \\ Yuan Nie (iD) \\ Xuan Zhu
}

Department of Gastroenterology, The First Affiliated Hospital of Nanchang University, Nanchang 330006, People's Republic of China

*These authors contributed equally to this work
Correspondence: Xuan Zhu Department of Gastroenterology, The First Affiliated Hospital of Nanchang University, No. 17, Yongwaizhengjie Road, Donghu District, Nanchang 330006. Jiangxi, People's Republic of China Email jyyfyzx@I63.com
Background: Acute-on-chronic liver failure (ACLF) is an extremely clinical entity associated with short-term high mortality. The CLIF-SOFA score measures both hepatic and extrahepatic organ dysfunction and can discriminate significantly better between survivors and nonsurvivors compared to other methods. The MELD score is widely used for organ allocation in liver transplantation. Recent reports indicate that the PWR is a potential biomarker for predicting clinical outcomes. The ALBI score is a new score model for evaluating the severity of liver dysfunction. We aimed to compare these prognosis models to predict short-term mortality in ACLF patients.

Methods: A retrospective analysis of 89 ACLF patients between 2015 and 2018 was performed. The receiver operating characteristic (ROC) curve was used to assess the power of four prognosis models for predicting 28-day mortality in patients with ACLF.

Results: The ALBI score, MELD score and CLIF-SOFA score were significantly higher, and the PWR was slightly lower in nonsurviving ACLF patients than in surviving patients. The MELD score and ALBI score were positively correlated with the CLIF-SOFA score, while the PWR was inversely related to the CLIF-SOFA score. The area under the ROC curves (AUROCS) of the CLIF-SOFA score, PWR, ALBI score and MELD score were 0.804, $0.759,0.710$ and 0.670 , respectively.

Conclusion: The CLIF-SOFA score, PWR and ALBI score can better predict 28-day mortality in ACLF patients, but the MELD score has worse predictability. The CLIFSOFA score is the best prognosis model among these models. PWR may be a simple and useful tool that can predict 28-day outcome.

Keywords: CLIF-SOFA score, platelet-to-white blood cell ratio, albumin-bilirubin score, acute-on-chronic liver failure, prognosis

\section{Introduction}

Acute-on-chronic liver failure (ACLF) is a clinical entity characterized by acute deterioration of a patient with chronic liver disease. ${ }^{1}$ Patients with ACLF develop one or more organ failures, and ACLF can be classified into 3 grades according to the number of organ failures (ACLF-1, 2, 3). ${ }^{2}$ Recently, a national study from the USA found that in patients with decompensated cirrhosis, one in four developed ACLF; interestingly, one in four patients with ACLF died within 28 days. ${ }^{3}$ Therefore, it is necessary to choose a precise prognosis model to identify and intervene in this population with corresponding treatment or transfer to a liver transplantation center. The chronic liver failure-sequential organ failure assessment 
(CLIF-SOFA) score is established by the CLIF consortium to evaluate the severity in ACLF patients. ${ }^{2}$ The CLIFSOFA score has been validated as useful in predicting outcomes in ACLF patients. ${ }^{4}$ The model of end-stage liver disease (MELD) score was first applied to predict survival in patients undergoing transjugular intrahepatic portosystemic shunts (TIPS). ${ }^{5}$ In addition, the MELD score has been broadly employed to predict the risk of mortality in patients with cirrhosis and serves as a predictor of survival in patients awaiting liver transplantation (LT). ${ }^{6}$ The platelet-to-white blood cell ratio (PWR) has been considered an index that could predict mortality in patients with cancer and cardiovascular disease. ${ }^{7,8}$ PWR would be an ideal marker for predicting outcomes in ACLF patients. The albumin-bilirubin (ALBI) score consists of two indicators: albumin and bilirubin, and it was originally established to assess the severity of liver dysfunction. ${ }^{9}$ Apart from this, ALBI has already been shown to well apply to predicting outcomes in primary biliary cirrhosis patients. ${ }^{10}$ To the best of our knowledge, there have been no studies comparing four prognostic models in ACLF patients. We performed a retrospective study to assess the discriminative abilities of CLIF-SOFA, MELD, PWR and ALBI in predicting 28-day mortality of patients with ACLF.

\section{Patients and Methods}

\section{Study Patients}

This was a retrospective cohort study, and consecutive hospitalized patients with ACLF were admitted to the Department of Gastroenterology, the First Affiliated
Hospital of Nanchang University in China, between January 2015 and December 2018. The definition of ACLF was based on the European Association for the Study of Liver (EASL) Consortium, and the CLIF-C OF score was used to define organ failure. Patients were included when they met the following criteria: (a) age $\geq 18$ and (b) diagnosis of cirrhosis and ACLF (defined by the EASL-CLIF Consortium). The exclusion criteria included (1) not meeting the ACLF diagnostic criteria of EASL-CLIF; (2) hepatocellular carcinoma; (3) history of liver transplantation; and (4) uncertain 28-day outcome. The study protocol conformed to the Declaration of Helsinki and was approved by the institutional ethics committee of the First Affiliated Hospital of Nanchang University (No. 2015-1206). Written informed consent was obtained from patients.

\section{Definitions}

Liver cirrhosis was diagnosed by liver biopsy or supported by imaging technologies (CT, MRI or ultrasound). ACLF was defined according to EASL-CLIF criteria, as described below: No ACLF: (1) no organ failure or (2) no hepatic encephalopathy and single "nonkidney" organ failure who had a serum creatinine level $<1.5 \mathrm{mg} / \mathrm{dL}$ or (3) single cerebral failure who had a serum creatinine level $<1.5 \mathrm{mg} / \mathrm{dL}$. ACLF grade 1: (1) single kidney failure or (2) single organ failure (coagulation, circulation, respiration, or liver) who had a serum creatinine level of $1.5-1.9 \mathrm{mg} / \mathrm{dL}$ and/or mild to moderate hepatic encephalopathy or (3) single cerebral failure who had a serum creatinine level of $1.5-1.9 \mathrm{mg} / \mathrm{dl}$. ACLF grade 2: two

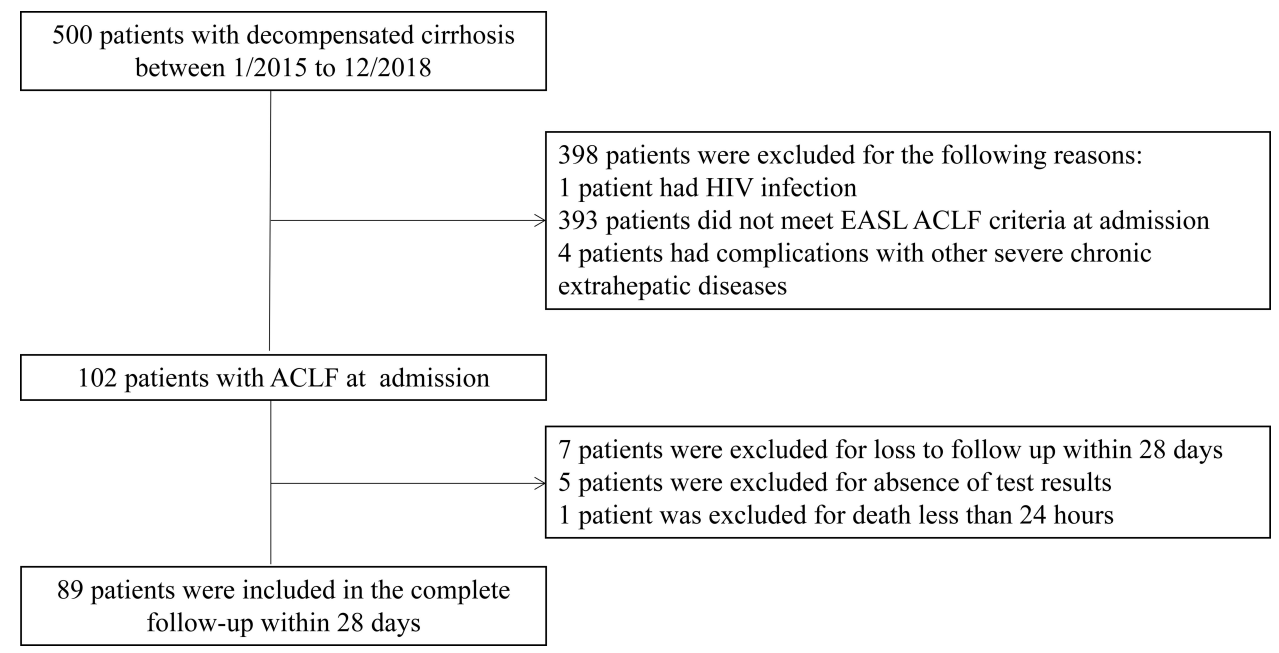

Figure I The flowchart in our study. 
organ failures. ACLF grade 3: three or more organ failures. The MELD score uses the patients' prothrombin time, INR, creatinine and serum bilirubin to predict survival and was calculated using the calculator: $11.2 \times \log$ $($ INR) $+3.8 \times \log$ (bilirubin $[\mathrm{mg} / \mathrm{dL}])+9.6 \times \log$ (creatinine $[\mathrm{mg} / \mathrm{dL}])+6.43 .{ }^{6}$ PWR was calculated by dividing platelets by white blood cells. ALBI was calculated from two variables, including bilirubin and albumin, and it was computed based on the formula: ALBI score $=0.66 \times \lg (\mathrm{TBiL}$ [umol/L])-0.085(albumin[g/L]). ${ }^{9}$

\section{Data Collection}

Laboratory and clinical data, demographics and the etiology of cirrhosis were collected from patients' clinical documents. ACLF grade was defined at the single time point when diagnosed with ACLF. All prognosis scores were calculated after collecting all data. Survival rates were obtained through patient electronic medical records or by direct contact with patients or their kin.

\section{Management of Patients}

Every patient received standard medical treatment in our study: patients with acute variceal bleeding (VB) received proton pump inhibitors, IV somatostatin and antibiotic prophylaxis. For those patients who failed to control the bleeding by drug therapy, urgent endoscopy hemostasis treatment was performed. Patients with bacterial infection were immediately treated with empirical antibiotic therapy, and the adjustment of antibiotic therapy was based on

Table I Clinical Characteristics of Study Participants

\begin{tabular}{|c|c|c|c|c|}
\hline & All Patients $(n=89)$ & 28-Day Survivors $(n=42)$ & 28-Day Nonsurvivors $(n=47)$ & P value \\
\hline Age, mean $\pm S D$ & $56.96 \pm 12.18$ & $57.33 \pm 12.53$ & $58.34 \pm \mid I .34$ & 0.687 \\
\hline Male sex $[\%(n)]$ & $66.3 \%(59)$ & $69.0 \%(29)$ & $63.8 \%(30)$ & 0.603 \\
\hline Hospitalization days, median (IQR) & $4(I-11)$ & $9(3.75-12.25)$ & $2(1-6)$ & $<0.001$ \\
\hline \multicolumn{5}{|l|}{ Aetiology of chronic liver disease } \\
\hline Hepatitis B Virus [\%(n)] & $41.6 \%(37)$ & $33.3 \%(14)$ & $48.9 \%(23)$ & 0.136 \\
\hline Alcoholic liver disease $[\%(n)]$ & $20.2 \%(18)$ & $21.4 \%(9)$ & $19.1 \%(9)$ & \\
\hline Hepatitis C Virus [\%(n)] & $1.9 \%(2)$ & $4.8 \%(2)$ & $0.0 \%(0)$ & \\
\hline Primary biliary cirrhosis [\%(n)] & $3.9 \%(4)$ & $7.1 \%(3)$ & $2.1 \%(1)$ & \\
\hline Others $[\%(n)]$ & $31.5 \%(28)$ & $33.3 \%(14)$ & $29.8 \%(14)$ & \\
\hline \multicolumn{5}{|l|}{ Primary reason for hospitalization } \\
\hline Variceal bleeding $[\%(n)]$ & $69.7 \%(62)$ & $66.7 \%(28)$ & $72.3 \%(34)$ & 0.561 \\
\hline Ascites $[\%(n)]$ & $5.6 \%(5)$ & $2.4 \%(1)$ & $8.5 \%(4)$ & \\
\hline Hepatic encephalopathy $[\%(n)]$ & $14.6 \%(13)$ & $16.7 \%(7)$ & $12.8 \%(6)$ & \\
\hline Infection $[\%(n)]$ & $9.0 \%(8)$ & $11.9 \%(5)$ & $6.4 \%(3)$ & \\
\hline Others $[\%(n)]$ & $0.9 \%(I)$ & $2.4 \%(1)$ & $0.0 \%(0)$ & \\
\hline \multicolumn{5}{|l|}{ ACLF grade } \\
\hline ACLF grade I [\%(n)] & $31.5 \%(28)$ & $54.8 \%(23)$ & $10.6 \%(5)$ & $<0.001$ \\
\hline ACLF grade $2[\%(n)]$ & $41.6 \%(37)$ & $35.7 \%(15)$ & $46.8 \%(22)$ & \\
\hline ACLF grade $3[\%(n)]$ & $26.9 \%(24)$ & $9.5 \%(4)$ & $42.6 \%(20)$ & $<0.001$ \\
\hline Endoscopic hemostasis [\%(n)] & $51.7 \%(46)$ & $57.1 \%(24)$ & $46.8 \%(22)$ & \\
\hline \multicolumn{5}{|l|}{ The degree of ascites } \\
\hline Mild [\%(n)] & $12.4 \%(\mathrm{II})$ & $14.3 \%(6)$ & $10.6 \%(5)$ & \\
\hline Moderate $[\%(n)]$ & $27.5 \%(25)$ & $28.6 \%(12)$ & $27.7 \%(13)$ & \\
\hline Severe $[\%(n)]$ & $24.5 \%(24)$ & $11.9 \%(5)$ & $40.4 \%(19)$ & 0.002 \\
\hline Artificial liver therapy [\%(n)] & $9.8 \%(9)$ & $4.8 \%(2)$ & $14.9 \%(7)$ & 0.114 \\
\hline Mechanical ventilation $[\%(n)]$ & $40.2 \%(37)$ & $9.5 \%(4)$ & $70.2 \%(33)$ & $<0.001$ \\
\hline Vasopressor use [\%(n)] & $65.2 \%(58)$ & $52.4 \%(22)$ & $76.6 \%(36)$ & 0.017 \\
\hline
\end{tabular}

Note: $P$ value $<0.05$ was considered significant and was indicated in bold.

Abbreviations: ACLF, acute-on-chronic liver failure; SD, standard deviation; IQR, interquartile range. 
antibiotic sensitivity test and bacteria culture. Decompensated cirrhosis resulting from hepatitis $\mathrm{B}$ virus infection was immediately given nucleoside analogs (entecavir alone $0.5 \mathrm{mg}$, telbivudine alone $600 \mathrm{mg}$, lamivudine alone $100 \mathrm{mg}$, or lamivudine $100 \mathrm{mg}$ plus adefovir $10 \mathrm{mg}$ daily). Patients with $\mathrm{HCV}$ virus infection were treated through direct-acting antiviral (DAA) treatment. Patients also received albumin and terlipressin, if required.

\section{Statistical Analysis}

The statistical analyses were performed using SPSS software version 20.0 (SPSS Inc., Chicago, IL). Continuous variables were expressed as the mean \pm standard deviation (SD) or medians (interquartile range $[\mathrm{IQR}]$ ), and categorical data were expressed as percentages. Differences in variables were analyzed using Student's $t$-tests or the Mann-Whitney $U$-test. Categorical data were evaluated by the chi-squared or Fisher exact test, as appropriate. The correlation between two variables was assessed using the Spearman rank correlation test. Receiver operating characteristic (ROC) curves were used to measure the performance of the score for the prediction of 28-day mortality in ACLF patients. Logistic regression analysis was performed to confirm the independent predictors for 28-day mortality in ACLF patients. The areas under the ROC curve (AUROCs) were compared using the DeLong test. All statistical testing was performed at the twotailed $\alpha$ level of 0.050 .

\section{Results}

\section{Baseline Characteristics of ACLF Patients}

A total of 102 ACLF patients were enrolled in this study between January 2015 and December 2018, and the flowchart is shown in Figure 1. During the study period, 89 patients were enrolled in the analysis of the outcomes at 28 days. The baseline characteristics are listed in Table 1. ACLF patients were divided into nonsurviving $(n=47)$ and surviving groups $(n=42)$ based on 28-day outcomes. No significant differences were observed for age and gender between the two groups, and the hospitalization days were significantly shorter in the nonsurviving groups. Hepatitis B virus (HBV) was the most prevalent cause of background liver disease (41.6\%). Sixty-two patients (69.7\%) were hospitalized due to variceal bleeding, which was the main reason for hospitalization. The ACLF grade was grade $2(37 / 89,41.6 \%)$, followed by grade $1(28 / 89,31.5 \%)$, followed by grade $3(24 / 89$, $26.9 \%)$. The degree of ascites was moderate $(25 / 89$,
$28.1 \%)$, followed by severe $(24 / 89,26.9 \%)$ and mild (11/ $89,12.4 \%)$. In the nonsurviving group, patients with ACLF grade 3, severe ascites, the use of mechanical ventilation and vasopressors were significantly higher than those in the surviving group. The leading causes of death at 28 days were hemorrhagic shock (15/47, 31.9\%), followed by respiratory failure $(12 / 47,25.5 \%)$, hepatic encephalopathy $(8 / 47,17.0 \%)$, infectious shock $(4 / 47$, $8.5 \%)$, liver failure $(4 / 47,8.5 \%)$, and uncertainty $(1 / 47$, $2.1 \%$ ). Supplement Table 1 depicts the cause of death at 28 days. Supplement Table 2 shows the specific number of organ failures in ACLF patients, there is no statistical significance between patients with and those without variceal bleeding events.

\section{Comparison of ALBI, PWR, MELD, CLIF-SOFA Scores and Clinical}

\section{Characteristics Between Nonsurviving and Surviving Acute-on-Chronic Liver Failure Patients}

The clinical characteristics between nonsurviving and surviving patients with ACLF are shown in Table 2. The nonsurviving patients had a higher CLIF-SOFA score, ALBI score, MELD score, bilirubin, INR, lactate, white blood cell count, ALT, AST, ALP, PT, and a lower PWR, platelet and albumin compared with surviving patients. These data indicate that the lower PWR in the nonsurviving group was due to an increased number of white blood cells and decreased platelets. No significant differences in creatinine, MAP, PO2/FiO2, GGT or BUN were detected.

\section{CLIF-SOFA Score and Platelet Were the Independent Risk Factors for 28-Day Mortality in Patients with ACLF}

In the univariate analysis for 28-day unfavorable prognosis, INR, platelet, Lactate, albumin, ALT, PT, PWR, CLIF-SOFA and ALBI were statistically significant because all had $\mathrm{P}$ values less than 0.05 , which is shown in Table 3. After adjusting the influences of the factors above by multivariate logistic regression, CLIF-SOFA score (OR:1.493 (1.149-1.940), $\mathrm{P}=0.016)$ and platelet (OR:0.982 (0.967-0.997), $\mathrm{P}=0.003$ ) were the independent predictor for unfavorable outcome. 
Table 2 Comparison of Clinical Characteristics and Prognostic Model Between Nonsurviving and Surviving Patients with ACLF

\begin{tabular}{|c|c|c|c|}
\hline Variables & Survivors(n=42) & $\begin{array}{l}\text { Nonsurvivors } \\
(n=47)\end{array}$ & P-value \\
\hline $\begin{array}{l}\text { Bilirubin, } \\
\mathrm{mmol} / \mathrm{L}\end{array}$ & $\begin{array}{l}34.50 \\
(15.38-69.00)\end{array}$ & $\begin{array}{l}58.10 \\
(26.60-137.80)\end{array}$ & 0.015 \\
\hline $\begin{array}{l}\text { Creatinine, } \\
\mathrm{mmol} / \mathrm{L}\end{array}$ & $\begin{array}{l}120.45 \\
(70.60-166.70)\end{array}$ & $\begin{array}{l}117.10 \\
(72.90-190.30)\end{array}$ & 0.560 \\
\hline INR & $1.52(1.31-1.74)$ & $1.74(1.52-2.68)$ & 0.008 \\
\hline Platelet, I0*9/L & $\begin{array}{l}76.50 \\
(35.75-139.00)\end{array}$ & $50.00(20.00-76.00)$ & 0.001 \\
\hline MAP, $\mathrm{mmHg}$ & $82.17 \pm|4.4|$ & $78.67 \pm 16.47$ & 0.290 \\
\hline $\mathrm{PO} 2 / \mathrm{FiO} 2$ & $342.26 \pm 129.44$ & $305.12 \pm 144.88$ & 0.208 \\
\hline Lactate, $\mathrm{mmol} / \mathrm{L}$ & $2.35(1.65-4.28)$ & $5.10(3.00-9.80)$ & $<0.001$ \\
\hline WBC, $10 * 9 / \mathrm{L}$ & $6.98(4.12-9.85)$ & $10.67(5.46-15.35)$ & 0.021 \\
\hline Albumin, $g / L$ & $26.91 \pm 5.30$ & $23.95 \pm 4.93$ & 0.008 \\
\hline ALT, IU/L & $26.5(\mid 8.0-56.25)$ & $48.0(25.0-188.0)$ & 0.021 \\
\hline AST, IU/L & $55.0(31.0-86.0)$ & $105.0(41.0-449.0)$ & 0.019 \\
\hline ALP, IU/L & $73.0(59.5-112.2)$ & $112.0(63.0-183.0)$ & 0.045 \\
\hline GGT, IU/L & $\begin{array}{l}35.50 \\
(15.00-165.75)\end{array}$ & $33.0(16.0-107.0)$ & 0.954 \\
\hline $\mathrm{BUN}, \mathrm{mmol} / \mathrm{L}$ & $10.95(6.65-18.52)$ & $14.10(8.40-19.80)$ & 0.121 \\
\hline PT & $\begin{array}{l}16.40 \\
(13.77-19.60)\end{array}$ & $21.20(16.70-26.00)$ & 0.002 \\
\hline ALBI score & $-1.259 \pm 0.538$ & $-0.850 \pm 0.533$ & 0.001 \\
\hline PWR & $10.86(5.73-20.17)$ & $4.19(1.96-7.83)$ & $<0.001$ \\
\hline MELD score & $16(13.75-20.00)$ & $24(15-29)$ & 0.006 \\
\hline $\begin{array}{l}\text { CLIF-SOFA } \\
\text { score }\end{array}$ & $8.57 \pm 2.71$ & $12.13 \pm 3.14$ & $<0.001$ \\
\hline
\end{tabular}

Note: $P$ value $<0.05$ was considered significant and was indicated in bold. Abbreviations: INR, international normalized ratio; MAP, mean arterial pressure; WBC, white blood cell count; ALT, alanine aminotransferase; AST, aspartate aminotransferase; ALP, alkaline phosphatase; GGT, gamma-glutamyl transpeptidase; BUN, blood urea nitrogen; PT, prothrombin time; ALBI, albumin-bilirubin; PWR, plateletto-white Blood Cell Ratio; MELD, model for end-stage liver disease; CLIF-SOFA, chronic liver failure-sequential organ failure assessment.

\section{ALBI Score, PWR and MELD Score are Correlated with the CLIF-SOFA Score}

The ALBI score and MELD score were found to be significantly positively correlated with the CLIF-SOFA score $(\mathrm{r}=0.430, \mathrm{r}=0.529$; both $P<0.001)$, and distinct negative correlations between the PWR and CLIF-SOFA score were detected in ACLF patients $(r=-0.330$; $P=0.002)$. Scatter diagrams of the ALBI score, MELD score and PWR are shown in Supplement Figures 1-3, respectively.

\section{Comparison of Four Predictive Models for Predicting 28-Day Mortality in ACLF Patients}

As shown in Figure 2, receiver operating characteristic curves (ROC) were applied to estimate the relative efficiencies of the ALBI score, PWR, MELD score and CLIFSOFA score for predicting 28-day mortality. The areas under the curve (AUC) values were 0.804 for the CLIFSOFA score, 0.759 for the PWR, 0.710 for the ALBI score and 0.670 for the MELD score. Further analysis was performed by comparing the AUC among the CLIF-SOFA score and the other three scores by the DeLong test. We found that the AUC of the CLIF-SOFA score was significantly higher than that of the MELD score $(P<0.05)$, but there was no difference between the ALBI score and PWR (both $P>0.05$ ). All these data analyses are listed in Table 4 .

\section{Discussion}

ACLF is a syndrome with a rapid downhill course and a poor short-term outcome. In this study of 89 ACLF patients with diverse etiologies, HBV was the predominant etiology, in line with the characteristics of the Asian population. ${ }^{11}$ It is worth noting that the overall mortality rate was $52.8 \%$ within 28 days, and the mortality rates in patients with ACLF grades 1, 2 and 3 were 17.8\%, 59.4\% and $83.3 \%$, respectively. In the analysis of data, we determined that the CLIF-SOFA score, ALBI score, and MELD score were statistically higher, and PWR was statistically lower in deceased patients. A significant positive association was found between ALBI score, MELD score and CLIF-SOFA score; conversely, PWR was negatively associated with CLIF-SOFA score. Additionally, the predictive power of the CLIF-SOFA score was significantly higher $(\mathrm{AUC}=0.804)$ than that of the MELD score $(\mathrm{AUC}=0.670$, $P<0.05$ ); however, when comparing the AUC of the CLIFSOFA score with PWR (AUC $=0.759$ ) and ALBI score (AUC $=0.710$ ), there was no significant difference between them $(P>0.05)$.

The risk of death was very high in our study, which was related to the severity of ACLF. Data from the EASL-CLIF Acute-on-Chronic Liver Failure in Cirrhosis (CANONIC) study confirmed that overall 28-day mortality was $33 \%$ of all cases of ACLF, and mortality increased with the degree of ACLF. ${ }^{1}$ Furthermore, the leading etiology was HBV, 
Table 3 Risk Factors Associated with 28-Day Mortality According to Logistic Regression Analysis

\begin{tabular}{|c|c|c|c|c|}
\hline & \multirow{2}{*}{$\begin{array}{l}\text { Univariate } \\
\text { OR }(95 \% \mathrm{CI})\end{array}$} & \multirow[b]{2}{*}{ P-value } & \multirow{2}{*}{$\begin{array}{l}\text { Multivariate } \\
\text { OR }(95 \% \mathrm{Cl})\end{array}$} & \multirow[b]{2}{*}{ P-value } \\
\hline & & & & \\
\hline \multicolumn{5}{|l|}{ Age } \\
\hline$\leq 50$ & Reference & & & \\
\hline$>50$ & $1.007(0.973-1.043)$ & 0.678 & & \\
\hline \multicolumn{5}{|l|}{ Sex } \\
\hline Woman & Reference & & & \\
\hline Man & $0.791(0.327-1.915)$ & 0.603 & & \\
\hline \multirow[t]{2}{*}{ Ever had HE } & Reference & & & \\
\hline & $0.852(0.369-1.970)$ & 0.708 & & \\
\hline Bilirubin, $\mathrm{mmol} / \mathrm{L}$ & $1.004(0.999-1.009)$ & 0.094 & & \\
\hline Creatinine, $\mathrm{mmol} / \mathrm{L}$ & $1.000(0.998-1.003)$ & 0.756 & & \\
\hline INR & $1.847(1.029-3.318)$ & 0.040 & & \\
\hline Platelet, I0*9/L & $0.981(0.970-0.992)$ & 0.001 & $0.982(0.967-0.997)$ & 0.016 \\
\hline MAP, $\mathrm{mmHg}$ & $0.985(0.958-1.013)$ & 0.289 & & \\
\hline $\mathrm{PO} 2 / \mathrm{FiO} 2$ & $0.998(0.995-1.001)$ & 0.207 & & \\
\hline Lactate, $\mathrm{mmol} / \mathrm{L}$ & $1.228(1.069-1.411)$ & 0.004 & & \\
\hline WBC, $10 * 9 / \mathrm{L}$ & $1.056(0.996-1.120)$ & 0.066 & & \\
\hline Albumin, $g / L$ & $0.891(0.815-0.974)$ & 0.011 & & \\
\hline ALT, IU/L & $1.003(1.000-1.006)$ & 0.045 & & \\
\hline AST, IU/L & $1.001(1.000-1.003)$ & 0.073 & & \\
\hline GGT, IU/L & $0.999(0.995-1.003)$ & 0.512 & & \\
\hline BUN, mmol/L & $1.015(0.977-1.054)$ & 0.451 & & \\
\hline $\mathrm{Na}, \mathrm{mmol} / \mathrm{L}$ & $1.051(0.993-1.113)$ & 0.087 & & \\
\hline PT & $1.064(1.004-1.127)$ & 0.037 & & \\
\hline PWR & $0.955(0.918-0.994)$ & 0.024 & & \\
\hline CLIF-SOFA & $1.488(1.245-1.780)$ & $<0.001$ & $1.493(1.149-1.940)$ & 0.003 \\
\hline ALBI & $4.274(1.747-10.456)$ & 0.001 & & \\
\hline
\end{tabular}

Notes: $P$ value $<0.05$ was considered significant and was indicated in bold. Data are expressed as the median (interquartile range).

Abbreviations: INR, international normalized ratio; MAP, mean arterial pressure; WBC, white blood cell count; ALT, alanine aminotransferase; AST, aspartate aminotransferase; ALP, alkaline phosphatase; GGT, gamma-glutamyl transpeptidase; BUN, blood urea nitrogen; PT, prothrombin activity; CTP, Child-Turcotte-Pugh; MELD, model for end-stage liver disease; MELD-Na, model for end-stage liver disease-sodium; CLIF-C OF, chronic liver failure consortium organ function; CLIF-SOFA, chronic liver failure-sequential organ failure assessment; CLIF-C ACLF, chronic liver failure consortium acute-on-chronic liver failure; NACSELD-ACLF, North American Consortium for the Study of End-Stage Liver Disease acute liver failure.

which was different from patients in Western countries. HBV-ACLF is a universal syndrome in Asia and Africa that is related to unacceptably high short-term mortality. ${ }^{12}$

As described above, the CANONIC study established the CLIF-SOFA score, which was useful for diagnosing ACLF according to the number and type of organ failure.
Based on our results, compared to other prognostic models, the CLIF-SOFA score performed better in predicting short-term mortality for ACLF. One retrospective study demonstrated that the CLIF-SOFA score outperformed other scoring systems in predicting short-term mortality for acutely decompensated alcoholic cirrhosis. ${ }^{13}$ In 


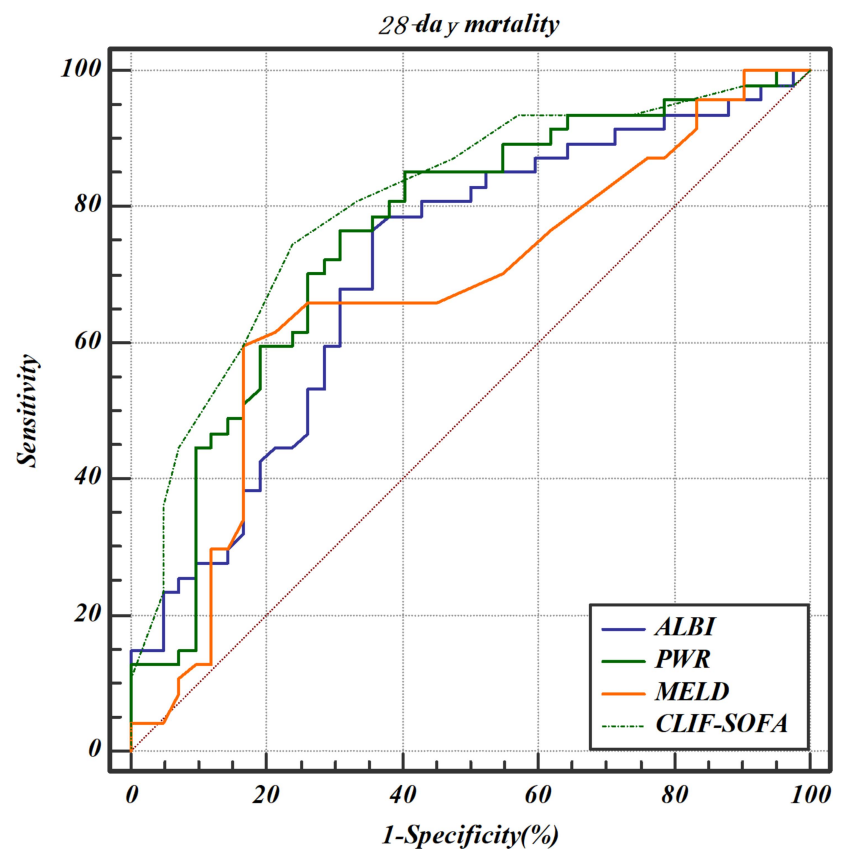

Figure 2 ROC curve analysis for predicting 28-day mortality by ALBI score, PWR, MELD and CLIF-SOFA score.

Abbreviations: ALBI, albumin-bilirubin; PWR, platelet-to-white blood cell ratio; MELD, model of end-stage liver disease; CLIF-SOFA, chronic liver failure-sequential organ failure assessment.

a separate study of patients with ACLF precipitated by either hepatic or extrahepatic insults, the CLIF-SOFA score was regarded as displaying the highest accuracy in predicting outcomes. ${ }^{14}$ The CLIF-SOFA score reflects multiorgan dysfunction, the ACLF patients with more organ failures, and the outcome became more terrible. ${ }^{15}$ However, although the CLIF-SOFA score had excellent predictive value, the calculation process was burdensome and onerous, which may limit clinicians' adhibition. Our analysis demonstrated that the MELD score had a lower predictive power than the other three scores. The MELD score did not contain the evaluation of subjective factors such as hepatic encephalopathy and ascites; it was composed of INR, TBIL and creatinine, which may reduce the accuracy of assessment. ${ }^{16}$

The ALBI score uses albumin and bilirubin levels to show liver damage, and it has been confirmed to be a useful score in reflecting the prognosis of hepatocellular carcinoma. ${ }^{17}$ Our data showed that the ALBI score and CLIF-SOFA score had similar efficacy in assessing the prognosis of ACLF patients. One single-center study indicated that the ALBI score was more effective and accurate than Child-Pugh and MELD scores in predicting the longterm prognosis for HBV-ACLF. ${ }^{18}$ A previous study found that a high ALBI score was an independent predictor of 3-month mortality in HBV-ACLF. ${ }^{19}$ The ALBI score may be expected to be widely clinically utilized as an efficient and simple scoring system.

PWR incorporated only two common hematological indexes, platelets and white blood cells, which made it easier and simpler to compute. In our analysis of prognostic outcomes, the data revealed that PWR had a predictive efficacy similar to CLIF-SOFA score in terms of predicting short-term mortality in ACLF patients. A recent study showed that PWR was an independent prognostic indicator in predicting outcomes of ACLF. ${ }^{20}$ The mechanisms still need to be investigated further, and one possibility is that the decreased PLT count and increased WBC count in ACLF patients. Low platelets are related to liver cirrhosis, and hypersplenism and portal hypertension can cause thrombocytopenia. ${ }^{21}$ An observational study found that the degree of lower platelets was associated

Table 4 The Predictive Value of Prognosis Models

\begin{tabular}{|l|l|l|l|l|l|}
\hline Prognostic Score & ROC Area (95\% CI) & P-value & Cut-Off Point & Sensitivity (\%) & Specificity (\%) \\
\hline ALBI & $0.710(0.604-0.801)$ & $<0.001$ & -1.12 & 76.60 & 64.29 \\
\hline PWR & $0.759(0.657-0.843)$ & $<0.001$ & 7.83 & 76.60 & 69.05 \\
\hline MELD & $0.670(0.562-0.766)$ & $\mathbf{0 . 0 0 4}$ & 21.00 & 59.57 & 74.47 \\
\hline CLIF-SOFA & $0.804(0.707-0.88 I)$ & $<0.001$ & 10.00 & & 76.19 \\
\hline CLIF-SOFA vs ALBI & $0.095(-0.031-0.221)$ & 0.141 & & & \\
\hline CLIF-SOFA vs PWR & $0.045(-0.082-0.173)$ & 0.485 & & & \\
\hline CLIF-SOFA vs MELD & $0.134(0.027-0.242)$ & $\mathbf{0 . 0 1 4}$ & & \\
\hline
\end{tabular}

Note: $P$ value $<0.05$ was considered significant and was indicated in bold.

Abbreviations: ROC, receiver operating characteristic; ALBI, albumin-bilirubin; PWR, platelet-to-white blood cell ratio; MELD, model for end-stage liver disease; CLIFSOFA, chronic liver failure-sequential organ failure assessment. 
with the severity of liver cirrhosis. ${ }^{22}$ In addition, the increased WBC count is associated with systemic inflammation, which was attributed to bacterial infection in ACLF patients. ${ }^{23}$

Several limitations existed in this study. First, this was a single-center and retrospective study, and the sample size was relatively small. Second, the laboratory data were not observed dynamically, which may cause bias. Finally, HBV was the leading etiology of these patients, which caused etiological bias.

In conclusion, we have found that the CLIF-SOFA score, PWR and ALBI score are all effective tools to predict 28-day mortality in ACLF patients; by contrast, the MELD score has a poor performance. The CLIF-SOFA score outperforms other prognostic models in terms of AUROC, but the evaluation is complicated; therefore, PWR may be a reliable and valuable tool to predict shortterm outcomes in ACLF.

\section{Ethical Approval}

This study was approved by the Ethics Committee of The First Affiliated Hospital of Nanchang University (No.2015-1206). Written informed consent was obtained from all participants. This study was conducted in accordance with the Declaration of Helsinki.

\section{Acknowledgments}

We would like to thanks the National Natural Science Foundation of China, the "Gan-Po Talent 555" Project of Jiangxi Province and the Postgraduate Innovation Special Foundation of Jiangxi Province for the economic support. LXL, YZ and YN are co-first authors in this manuscript and contributed equally to this study.

\section{Author Contributions}

All authors made a significant contribution to the work reported, whether that is in the conception, study design, execution, acquisition of data, analysis and interpretation, or in all these areas; took part in drafting, revising or critically reviewing the article; gave final approval of the version to be published; have agreed on the journal to which the article has been submitted; and agree to be accountable for all aspects of the work.

\section{Funding}

This study was supported by the National Natural Science Foundation of China (grant number: 81960120), "Gan-Po Talent 555" Project of Jiangxi Province (GCZ (2012)-1) and the Postgraduate Innovation Special Foundation of Jiangxi Province (YC2020-B046).

\section{Disclosure}

The authors declare that there are no conflicts of interest.

\section{References}

1. Moreau R, Jalan R, Gines P, et al. Acute-on-chronic liver failure is a distinct syndrome that develops in patients with acute decompensation of cirrhosis. Gastroenterology. 2013;144(7):1426-37, 37 e1-9. doi:10.1053/j.gastro.2013.02.042

2. Jalan R, Saliba F, Pavesi M, et al. Development and validation of a prognostic score to predict mortality in patients with acute-onchronic liver failure. J Hepatol. 2014;61(5):1038-1047. doi:10.1016/ j.jhep.2014.06.012

3. Hernaez R, Kramer JR, Liu Y, et al. Prevalence and short-term mortality of acute-on-chronic liver failure: a national cohort study from the USA. J Hepatol. 2019;70(4):639-647. doi:10.1016/j. jhep.2018.12.018

4. Song DS, Kim TY, Kim DJ, et al. Validation of prognostic scores to predict short-term mortality in patients with acute-on-chronic liver failure. J Gastroenterol Hepatol. 2018;33(4):900-909. doi:10.1111/jgh.13991

5. Malinchoc M, Kamath PS, Gordon FD, Peine CJ, Rank J, Ter Borg PC. A model to predict poor survival in patients undergoing transjugular intrahepatic portosystemic shunts. Hepatology. 2000;31(4):864-871.

6. Kamath PS, Wiesner RH, Malinchoc M, et al. A model to predict survival in patients with end-stage liver disease. Hepatology. 2001;33 (2):464-470. doi:10.1053/jhep.2001.22172

7. Cicek G, Acikgoz SK, Yayla C, Kundi H, Ileri M. White blood cell count to mean platelet volume ratio: a novel and promising prognostic marker for ST-segment elevation myocardial infarction. Cardiol J. 2016;23(3):225-235. doi:10.5603/CJ.a2016.0001

8. Garbens A, Wallis CJD, Bjarnason G, et al. Platelet to white blood cell ratio predicts 30 -day postoperative infectious complications in patients undergoing radical nephrectomy for renal malignancy. Can Urol Assoc J. 2017;11(11):E414-E20. doi:10.5489/cuaj.4478

9. Johnson PJ, Berhane S, Kagebayashi C, et al. Assessment of liver function in patients with hepatocellular carcinoma: a new evidence-based approach-the ALBI grade. J Clin Oncol. 2015;33 (6):550-558. doi:10.1200/JCO.2014.57.9151

10. Chan AW, Chan RC, Wong GL, et al. New simple prognostic score for primary biliary cirrhosis: albumin-bilirubin score. $J$ Gastroenterol Hepatol. 2015;30(9):1391-1396. doi:10.1111/jgh.12938

11. Yuen MF, Yuan HJ, Wong DK, et al. Prognostic determinants for chronic hepatitis B in Asians: therapeutic implications. Gut. 2005;54 (11):1610-1614. doi:10.1136/gut.2005.065136

12. Li H, Chen LY, Zhang NN, et al. Characteristics, diagnosis and prognosis of acute-on-chronic liver failure in cirrhosis associated to hepatitis B. Sci Rep. 2016;6:25487. doi:10.1038/srep25487

13. Lee M, Lee JH, Oh S, et al. CLIF-SOFA scoring system accurately predicts short-term mortality in acutely decompensated patients with alcoholic cirrhosis: a retrospective analysis. Liver Int. 2015;35 (1):46-57. doi:10.1111/liv.12683

14. Maipang K, Potranun P, Chainuvati S, et al. Validation of the prognostic models in acute-on-chronic liver failure precipitated by hepatic and extrahepatic insults. PLoS One. 2019;14(7):e0219516.

15. Silva PE, Fayad L, Lazzarotto C, et al. Single-centre validation of the EASL-CLIF consortium definition of acute-on-chronic liver failure and CLIF-SOFA for prediction of mortality in cirrhosis. Liver Int. 2015;35(5):1516-1523. doi:10.1111/liv.12597

16. Durand F, Valla D. Assessment of prognosis of cirrhosis. Semin Liver Dis. 2008;28(1):110-122. doi:10.1055/s-2008-1040325 
17. Antkowiak M, Gabr A, Das A, et al. Prognostic role of albumin, bilirubin, and ALBI scores: analysis of 1000 patients with hepatocellular carcinoma undergoing radioembolization. Cancers. 2019;11 (6):879. doi:10.3390/cancers 11060879

18. Chen RC, Cai YJ, Wu JM, et al. Usefulness of albumin-bilirubin grade for evaluation of long-term prognosis for hepatitis B-related cirrhosis. J Viral Hepat. 2017;24(3):238-245. doi:10.1111/jvh.12638

19. Chen B, Lin S. Albumin-bilirubin (ALBI) score at admission predicts possible outcomes in patients with acute-on-chronic liver failure. Medicine. 2017;96(24):e7142. doi:10.1097/MD.0000000000007142

20. Jie Y, Gong J, Xiao C, et al. Low platelet to white blood cell ratio indicates poor prognosis for acute-on-chronic liver failure. Biomed Res Int. 2018;2018:7394904. doi:10.1155/2018/7394904
21. Pradella P, Bonetto S, Turchetto S, et al. Platelet production and destruction in liver cirrhosis. J Hepatol. 2011;54(5):894-900. doi:10.1016/j.jhep.2010.08.018

22. Vinholt PJ, Hvas AM, Nielsen C, et al. Reduced platelet activation and platelet aggregation in patients with alcoholic liver cirrhosis. Platelets. 2018;29(5):520-527. doi:10.1080/09537104.2017.1349308

23. Hernaez R, Sola E, Moreau R, Gines P. Acute-on-chronic liver failure: an update. Gut. 2017;66(3):541-553. doi:10.1136/gutjnl-2016312670

\section{Publish your work in this journal}

Risk Management and Healthcare Policy is an international, peerreviewed, open access journal focusing on all aspects of public health, policy, and preventative measures to promote good health and improve morbidity and mortality in the population. The journal welcomes submitted papers covering original research, basic science, clinical \& epidemiological studies, reviews and evaluations, guidelines, expert opinion and commentary, case reports and extended reports. The manuscript management system is completely online and includes a very quick and fair peer-review system, which is all easy to use. Visit http://www.dovepress.com/testimonials.php to read real quotes from published authors.

Submit your manuscript here: https://www.dovepress.com/risk-management-and-healthcare-policy-journal 